\title{
Die Schönburgische Landesschule in Geringswalde
}

\author{
Jörg Heinrich
}

Am 3. Juli 2016 jährte sich zum 450. Male die Gründung der Schönburgischen Landesschule in Geringswalde, deren trauriges Geschick sich nach nur zwei Jahren mit ihrer gewaltsamen Auflösung durch Kurfürst August von Sachsen (1526-1586) im Juli 1568 vollendete. Daran möchte dieser Beitrag erinnern.

In der Mitte des 16. Jahrhunderts befand sich im mittleren Süden des damaligen sächsischen Territoriums die reichsunmittelbare Herrschaft Schönburg, die nach 1559 aus der niederen Grafschaft Hartenstein, den Herrschaften Lichtenstein, Glauchau und Waldenburg sowie den kursächsischen Ämtern Remse, Penig, Rochsburg und Wechselburg bestand. Das Adelsgeschlecht von Schönburg war bereits in die Linien Glauchau, Waldenburg und Penig gegliedert, obgleich es eine Regierung des Gesamthauses Schönburg gab. Die Linien wurden von ihren Begründern, den Brüdern Georg, Hugo und Wolf von Schönburg, repräsentiert. Da die Linie Glauchau an der Schulgründung nicht beteiligt und der Errichtung einer Schule sehr zugetane Hugo von Schönburg bereits am 4. Februar 1566 verstorben war, wird hier allein Wolf von Schönburg (1532-1581) von Interesse sein.

Der Protestantismus hatte sich in der Herrschaft Schönburg mit dem Einzug der Reformation im Jahr 1542 durchgesetzt. Die erste evangelische Predigt war am 18. Oktober in Glauchau aus dem Munde des Reformators und Leipziger Superintendenten Johann Pfeffinger (1493-1573) zu vernehmen. Die Einrichtung einer Superintendentur in Glauchau erfolgte noch im gleichen Jahr, wenig später in Penig (1556) und Waldenburg (1559). Die Superintendenten von Penig, Magister Bartholomäus Rosinus (1520-1586), und Waldenburg, Christophorus Hoffmann, waren es dann auch, die ihre Herren für die Idee gewinnen konnten, nach den Vorbildern in Pforta, Meißen und Grimma auch in ihrer Herrschaft eine eigene Landesschule einzurichten. Die Wahl fiel dabei nicht zufällig auf Geringswalde, einem Städtchen der Rochlitzer Pflege. Hier befand sich mit dem St. Marienstift ein säkularisiertes Benediktinerinnenkloster, dessen Güter und Einkünfte künftig dem Unterhalt der Schule dienen sollten. Die beiden Superintendenten be- riefen Magister Hieronymus Haubold (15351579) aus Frankenberg und Jacob Mehlhorn aus Langenleuba bei Altenburg als Lehrkräfte an die Schule; ersteren als Rektor, letzteren als Kantor und Kollegen. Damit waren zwei überzeugungstreue wie willensstarke Lutheraner aus der Anhängerschaft des Matthias Flacius gewonnen; beide waren geradezu typische Vertreter des evangelischen Gelehrtenstandes der Mitte des 16. Jahrhunderts. Haubold hatte in Wittenberg studiert und dort den akademischen Grad eines Magisters erworben. Bevor er nach Geringswalde kam, war er Professor der Artistenfakultät in Greifswald. Der Bildungsweg Mehlhorns liegt im Dunkeln.

Am 3. Juli 1566 - auf den Tag 23 Jahre nach der Eröffnung der Fürstenschule St. Afra in Meißen - wurden die beiden Lehrer und die ersten zwölf Schüler in Geringswalde eingewiesen. Tags darauf fanden die Einweihungsfeierlichkeiten statt. Tüchtige Pfarrer und Streiter für das lutherische Christentum - orthodoxer, flacianischer Prägung selbstredend - sollte die Schule hervorbringen, wenn auch nicht ausschließlich. Bereits drei Monate später wurde die Schar der Schüler um fünfzehn Knaben erweitert. Es war ein hoffnungsvoller Anfang.

Die Gründung der Schule aber fiel in eine Zeit heftiger theologischer und weltanschaulicher Auseinandersetzungen. Insbesondere die junge protestantische Christenheit zerfiel in viele, sich oftmals unversöhnlich gegenüberstehende Strömungen. Überzeugt von der Richtigkeit und der Schlagkraft der eigenen Argumente, trugen die Kontrahenten den Kampf um die Rechtmäßigkeit der vertretenen Anschauung verbissen und unnachgiebig aus. Das Verhängnis der Schule

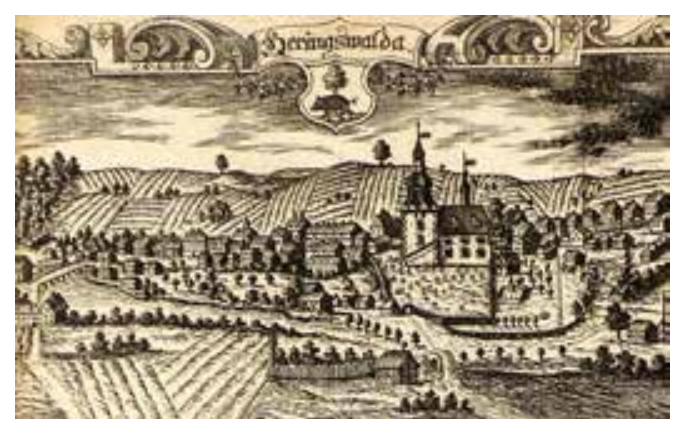

Dieser Beitrag beruht auf Theodor Distels Schrift „Der Flacianismus und die Schönburg'sche Landesschule zu Geringswalde", die 1879 in Leipzig erschienen ist. Distel selbst schöpfte aus den Akten des Sächsischen Staatsarchivs, aus Sekundärliteratur und aus der handschriftlichen Schulchronik des Rektors Hieronymus Haubold (1535-1579).

Geringswalde, Ansicht, um 1775 
rührte denn auch von den theologischen Zwistigkeiten dieser so streitgeneigten Zeit. 1560 hatten die Philippisten, die Parteigänger des Reformators Philipp Melanchthon, durch ein kurfürstliches Mandat vom 20. März, das den Corpus doctrinae christianae für die Geistlichen des Landes verbindlich erklärte, die Oberhand in den kirchlich-politischen Verhältnissen Kursachsens gewonnen. Durch das Mandat sahen sich vornehmlich zwei Geistliche der Herrschaft Schönburg, der Glauchauer Superintendent Magister Nikolaus Beheim und der Peniger Archidiakon Magister Bartholomäus Wagner, in ihrer Glaubensfreiheit beeinträchtigt. Sie unterrichteten daraufhin ihren Patron Wolf von Schönburg, weshalb sie dem kurfürstlichen Befehl nicht gehorchen konnten, und ersuchten ihn, weiteres $\mathrm{zu}$ veranlassen. Wolf hatte noch nichts unternommen, als der Peniger Rat und später die gesamte Geistlichkeit bereits vor das Leipziger Konsistorium geladen wurden. Dieses war durch den Diakon Kaspar Pennig heimlich von den Vorgängen in Kenntnis gesetzt worden. Wolf

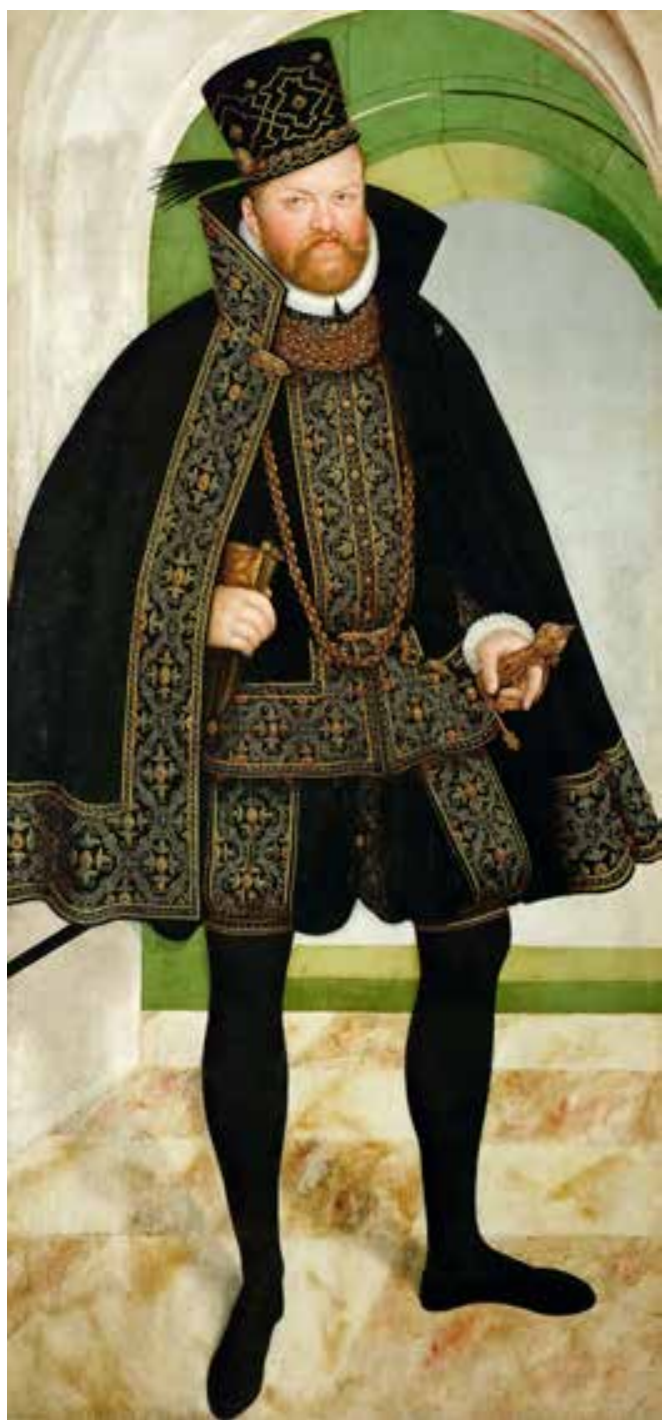

ließ sich dort vertreten und seine Abwesenheit entschuldigen. Das Konsistorium untersagte Beheim und Wagner das Predigen und Beichtesitzen, gestattete ihnen aber, das Evangelium zu lesen, die Passion zu singen, die Sakramente zu reichen und zu taufen. Der Patron wurde gewarnt, sich nicht persönlich in die Angelegenheit einzumischen, obgleich man ihn bei der Ausübung des Patronatsrechts nicht beeinträchtigen wolle. Beschwerden und Bitten an den Kurfürsten erwirken nichts: Das ganze Land habe das Corpus doctrinae angenommen. Ausnahmen könnten daher nicht gemacht werden, so der Kurfürst persönlich zu Wolf von Schönburg. Schließlich mussten Beheim und Wagner Kursachsen binnen Monatsfrist verlassen. Das Konsistorium erhielt Befehl, die durch den Patron neu anzustellenden Prediger einer gebührlichen Prüfung zu unterziehen, nachdem Wolf gegenüber dem Konsistorium geäußert hatte, er werde die Stellen doch wieder mit Männern besetzen, die der flacianischen Partei zugetan seien. Das war das Vorspiel!

Ein weiteres Mandat vom 18. Juni 1566 löste allgemeineren und heftigeren Widerstand aus. Wolf von Schönburg und seine Geistlichen zu Penig, einschließlich des Superintendenten Magister Christophorus Hoffmann, baten den Kurfürsten wegen des Mandats um Aufklärung und ließen diesen bereits Bedenken gegen dasselbe durchfühlen. August sprach den Ungehorsamen sein ernstes Missfallen aus und gab sich der Hoffnung hin, man werde sich fügen. Dies geschah nicht. Vielmehr antwortete die Gegenseite abermals mit einem Schreiben, in welchem sie dem Kurfürsten ihre Beweggründe lang auseinandersetzte. Diesem kam die Geduld abhanden. An das Leipziger Konsistorium erging der Befehl, die Geistlichen vorzuladen, um sie, im Fall des Ungehorsams, abzusetzen und der Herrschaft andere, friedliebendere Leute zu verordnen. Ein drittes Schreiben der Gegenseite gelangte noch an den Kurfürsten. Bei Strafe von 6.000 Gulden, so Augusts Antwort darauf, solle Wolf binnen Monatsfrist den Gehorsam seiner Prediger oder die erfolgte Neuanstellung melden. Umsonst! Das Konsistorium konnte nur berichten, dass die dort vorstellig gewordenen Prediger auf dem Flacianismus beharrten und deshalb bereits neue Prediger nach Penig gewiesen seien. Denen begegnete man dort wie zu erwarten abweisend und unhöflich. Wolf drohte ihnen sogar für den Fall, dass sie sich unterstehen würden zu predigen. So wagte keiner von ihnen, die Kanzel zu betreten. Dies erzürnte den Kurfürsten nicht wenig. Als Wolf in einem weiteren Schreiben an das Konsistorium von der Entziehung des Patronatsrechts sprach, drohte 


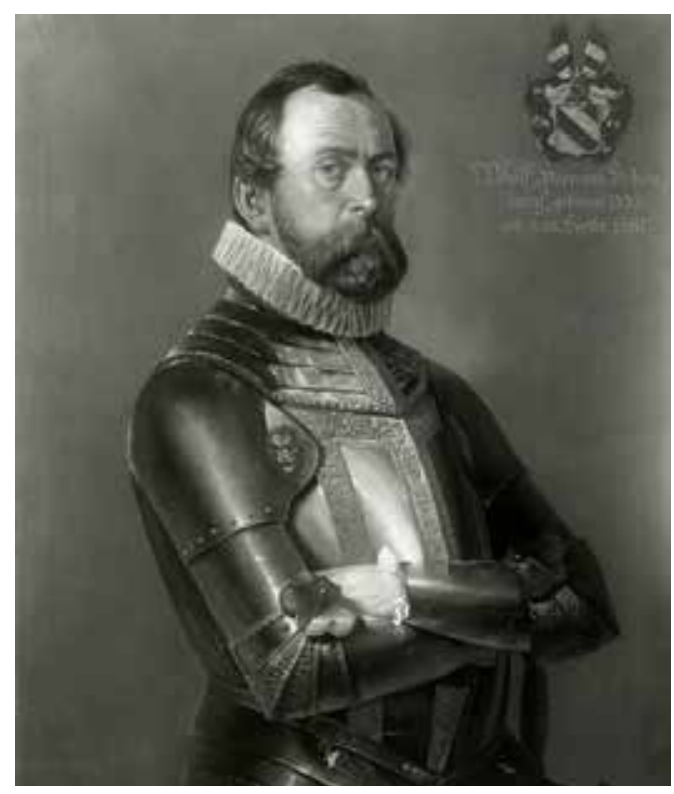

ihm der Kurfürst, ihn bei weiterer Verweigerung als seinen Lehnsmann und Untertanen nicht länger anerkennen zu wollen. Auch der Peniger Rat fiel in Ungnade. Bei Androhung von 4.000 Gulden Strafe wurde ihm die Annahme der neuen Prediger befohlen, was dann auch geschah. Wolf hingegen zeigte sich weiter standhaft und geißelte das kurfürstliche Mandat unchristlich. Er betonte, dass die ganze Angelegenheit nicht vor die weltliche Obrigkeit gehöre, sondern Gottes Sache sei, er also auch nicht zu gehorchen brauche und nicht gehorchen könne. Dies wollte August nicht auf sich beruhen lassen.

Das Vorgehen gegen Wolf geschah vorerst jedoch vorsichtig. Auf Zureden seiner Räte wurde zunächst die Superintendentur Penig aufgehoben und weiter Geistliche vor das Konsistorium zitiert. Eingedenk der Bedeutung der Angelegenheit empfahlen die Räte dem Kurfürsten, nicht sofort zum Äußersten zu schreiten, sondern Wolf weitere zwei Monate zur „Einkehr“ zuzugestehen. Am 7. März 1567 erging dann ein energisches kurfürstliches Schreiben, in dem Wolf wegen seines Ungehorsams an Stelle der vorher auferlegten Lehnsemende (Geldbuße) nunmehr die härteste Strafe seiner Felonie, der Verlust des Lehns selbst, angedroht wurde. Damit nicht genug. August wies die Schösser von Rochlitz und Chemnitz an, auszuforschen, wo sich Wolf regelmäßig aufhielt, um ihn gefangen zu nehmen. Die Bürgermeister von Zwickau, Freiberg, Annaberg, Marienberg, Leipzig, Dresden, Chemnitz und Schneeberg wies er an, Wolf sofort zu verhaften, sobald man seiner habhaft werde.

Dies geschah schon bald. Am 13. März 1567 wurde Wolf in Chemnitz „bestrickt“. Er wurde nach Dresden in die Schönburgische Behausung verbracht und durfte diese nicht ohne Genehmigung verlassen. Bis zur ersten Vernehmung am 21. Juni 1567 verfloss ein Vierteljahr. Wolf sollte dort beweisen, dass die kurfürstlichen Mandate gottlos und unchristlich wären. Er bat sich dazu Bedenkzeit bis zum nächsten Morgen aus. Diese gewährte man ihm nicht, vielmehr bestand man auf einer sofortigen Erklärung. Wolf mäßigte sich, bat um Verzeihung und hob hervor, dass er die Mandate für seine Person annehmen wolle. Dies genügte dem Kurfürsten nicht. Selbst die flehentlichen Bitten von Wolfs Gattin konnten sein Herz nicht erweichen. Wolf wurde in den Kerker unter dem Schlosshaupttor geworfen. Rasch verbreitete sich die Kunde davon und alsbald verwendeten sich zahlreiche Fürsprecher Wolfs beim Kurfürsten. Daraufhin befahl August am 25. Juni 1567, den Gefangenen in die Schösserei zu bringen, ihn dort eine vorgefertigte Abbitte sowie weitere Dokumente unterzeichnen zu lassen und ihn anschließend zu entlassen. Wolf wollte dies jedoch aus Gewissensgründen nicht in Gänze tun, und so zog sich das Hin und Her zwischen seinen Fürsprechern und dem Kurfürsten über Monate hin. Wolfs Gesundheit verschlechterte sich in dem elenden Kerker zusehends und am 24. Juli 1567 stand zu befürchten, er werde die Nacht nicht überleben. Nicht einmal dies rührte August. Wenn Wolf versterbe, solle ihm kein christliches Begräbnis zuteilwerden, ließ er sich vernehmen. Hier waren zwei harte Kontrahenten aneinandergeraten, von denen keiner nachgeben wollte. Mit nur kurzen Unterbrechungen saß Wolf bis zum 16. Oktober 1567, an welchem Tage er die Dokumente endlich unterzeichnete, im Kerker.

In Geringswalde hatte man unterdessen am 4. Juli 1567 den ersten Jahrestag der Schuleinweihung gefeiert und zugleich für Wolf gebetet: „Gib uns wieder unseren lieben Herrn, deinen frommen Knecht und beständigen Diener.“ Bei seiner Rückkehr im Spätherbst sammelte sich die Schule zu einem Dankgebet, welches ebenfalls Haubolds Feder entstammte. Doch seine Zeit in Geringswalde sollte nicht mehr lange währen. Schon Weihnachten 1566 hatte der Hofprediger Magister Christian Schütz dem Kurfürsten berichtet, dass in Geringswalde eine „urflacianische“ Schule bestehe, welche einer Visitation unterzogen werden müsse. Am 12. Juni 1568 befahl der Kurfürst die Durchführung der selbigen, und am 11. Juli 1568 wurde die Schule von den Visitatoren Joachim Camerarius (Melanchtons Freund), Doktor Andreas Freyhub, Magister Leonhard Lycius, Magister Balthasar Gütler, Doktor Caspar Peucer (Melanchtons Schwiegersohn) und Magister Heinrich Moller aufgesucht. Sie berichteten dem Kurfürsten tags
Wolf II. von Schönburg, Ölgemälde, unbekannter Maler ๑ SLUB Dresden, Deutsche Fotothek 
darauf und klagten über schwere große Gebrechen, die sie in der Schule vorgefunden hätten. Sie fügten dem Bericht ein Buch bei, welches sie unter vielen erwischt hatten. Es war eines der Argumentbücher, die Haubold den Schülern gegeben hatte. Haubold selbst schildert in seiner Schulchronik, dass die Visitatoren unangekündigt erschienen seien und zu erfahren begehrt hätten, wie es der Rektor in der Schule halte. Er habe eingewandt, dass es ihm schwerfalle, ohne Rücksprache mit seinem Herrn etwas in dieser Sache zu tun. Da jedoch ein kurfürstlicher Befehl vorliege und die Schule nichts zu verbergen habe, wolle er sich nicht widersetzlich zeigen. Die Visitatoren ließen sich Unterlagen zeigen und begannen anschließend dem Rektor verbal zuzusetzen. Es entspann sich letztendlich ein theologischer Disput um die rechte Lehre, in dem sich Haubold achtbar gegen die zahlenmäßige Überlegenheit der Visitatoren hielt.

Eine reichliche Woche später spitzten sich die Ereignisse zu. Am 20. Juli 1568 wurde von Colditz aus, wo der Kurfürst lagerte, ein Kundschafter in das Kloster Geringswalde geschickt, um auszuforschen, ob Haubold vor Ort wäre. Als Bettelstudent getarnt, bat er den Rektor um ein paar Pfennige. Dieser jedoch erkannte die List und war nun im Bilde. In der Nacht des 23. Juli 1568 verließ er nach einer Warnung heimlich das Kloster und wandte sich zu seinen Schwiegereltern nach Mittweida, wo seine hochschwangere Frau ihn erwartete. Als der Morgen dämmerte, schickte er seinen Neffen und Schüler Israel Mäusel nach Geringswalde, um die Lage auszukundschaften. Dieser fand das Kloster von 200 Mann umringt, angeführt von den Schössern zu Colditz und Leisnig. Haubold wurde mit großem Ernst gesucht und jeder Schüler zu seinem Verbleib ausführlich befragt. Da man seiner in Geringswalde nicht habhaft werden konnte, ritt der Colditzer Schösser nach Mittweida und traf auf halbem Wege Haubolds Neffen. Dieser gab sich als ein anderer aus und kehrte schleunigst nach Mittweida zurück. Er konnte Haubold knapp warnen, der sich zunächst in einem nahen Bauerngut verbarg, bis ihm ein Pferd gebracht wurde und er außer Landes fliehen konnte. An seiner statt hielt man sich an Kantor Mehlhorn schadlos und führte ihn in das Schloss Leisnig. Der Kurfürst überlegte nach der Lektüre des Visitationsberichts, ob er Wolf von Schönburg, da dieser offenbar gegen die Obligation gehandelt und sich das Visitationsrecht über die Schule zu Geringswalde angemaßt hatte, erneut nach Dresden fordern, dessen Schule abschaffen und dessen kursächsische Lehen einziehen sollte. Nachdem aber am 27. Juli 1568 auch der Schulverwalter Wiedemann, der die Schüler tags zuvor, nicht ohne ihnen ein Abschiedsessen auszurichten, nach Hause geschickt hatte, verhaftet worden war und die Einkünfte Wolfs aus dem Kloster beschlagnahmt worden waren, sandte August, auf Vorschlag seiner Räte, lediglich eine Kommission an Wolf ab. Sie sprach am 18. August 1568 bei ihm in Penig vor und brachte eine Erklärung Wolfs zu seiner Schule in Geringswalde mit nach Dresden. August nahm diese hin, ließ Wolf durch seine Räte gleichwohl wissen, dass nichts vergessen sei und bei kleinen oder großen Verstößen gegen die Obligation mitgestraft werden würde. Damit nun die Schule, an sich ein löbliches und gutes Werk, nicht etwa eingehen möge, befahl der Kurfürst dem Leipziger Konsistorium, dafür zu sorgen, dass die Knaben wieder dahin gebracht und andere Lehrer daselbst angestellt würden. Mit dieser Order und mit der Fortsetzung der Schule waren aber die Herren von Schönburg nicht einverstanden. Zwar war mit Wolfgang Dreifuss bereits ein neuer Rektor nach Geringswalde abgeschickt worden, doch musste derselbe unverrichteter Dinge wieder abziehen. Damit war der letzte Vorhang für die Schule gefallen.

Haubolds Flucht führt ihn zunächst zu Bartholomäus Rosinus nach Weimar, wo er den besonderen Schutz Herzog Johann Wilhelms genoss. Später zog er weiter nach Regensburg und Klagenfurt, wo er wiederum als Schulrektor fungierte, um schlussendlich ins Predigtamt nach Eferding in Oberösterreich zu gehen. Dort starb er am 15. Juni 1579. Mehlhorn wurde nach seinem Verhör am 3. August 1568 in den Turm zu Rochlitz geworfen und kam erst nach vielfachen, aber vergeblichen Bitten der Angehörigen am 13. März 1569 auf Fürsprache des Herzogs Johann Wilhelm wieder frei. 1582 finden wir auch ihn in Eferding. Einer seiner Geringswalder Schüler, Paulus Preuser, hatte dort Anstellung im Predigtamte gefunden. Mehlhorn selbst hatte nicht das Glück, die Stelle, die ihm dort in Aussicht gestellt worden war, zu erhalten. Sein weiterer Lebensweg lässt sich nicht mehr verfolgen.

War eingangs vom traurigen Geschick der Schule die Rede, trifft dies umso mehr zu, als sich die Verhältnisse in Kursachsen 1574, also nur sechs Jahre nach der gewaltsamen Auflösung der Schule, grundlegend änderten. Die Philippisten fielen in Augusts Ungnade und sahen sich nun selbst verfolgt. Die Flacianer, die uns in der Literatur auch als Gnesiolutheraner begegnen, wurden rehabilitiert und gaben fortan den Ton in den kirchlich-politischen Verhältnissen Kursachsens an. Welche Zukunft hätte die Geringswalder Schule unter diesen Umständen haben können? 